Port Acadie

Revue interdisciplinaire en études acadiennes

An Interdisciplinary Review in Acadian Studies

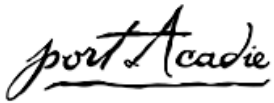

\title{
Repérage de quelques obstacles linguistiques et culturels des soins de santé pour les immigrants francophones de la Nouvelle-Écosse
}

\section{Louise Fontaine}

Numéro 18-19, automne 2010, printemps 2011

URI : https://id.erudit.org/iderudit/1010301ar

DOI : https://doi.org/10.7202/1010301ar

Aller au sommaire du numéro

\section{Éditeur(s)}

Université Sainte-Anne

ISSN

1498-7651 (imprimé)

1916-7334 (numérique)

Découvrir la revue

Citer cet article

Fontaine, L. (2010). Repérage de quelques obstacles linguistiques et culturels des soins de santé pour les immigrants francophones de la Nouvelle-Écosse. Port Acadie, (18-19), 103-115. https://doi.org/10.7202/1010301ar

\section{Résumé de l'article}

Ce texte s'attarde au concept d'« immigrant francophone » appliqué au contexte de la Nouvelle-Écosse. Contrairement à ce qu’on peut imaginer à un premier niveau d'analyse, ce concept est polysémique et le sens qu'on y attribue varie selon les acteurs sociaux qui l'utilisent. Donc, afin de mieux délimiter l'objet de notre propos, nous avançons quelques éléments de définition à prendre en compte. Il s'agit d'une recherche exploratoire orientée vers la formulation de quelques pistes de réflexion, voire quelques hypothèses de travail à vérifier lors de la réalisation de futures recherches dans le domaine de la santé auprès des communautés francophones et acadiennes de la Nouvelle-Écosse. Pour étoffer notre propos, nous présentons quelques données empiriques recueillies à même le travail de terrain mené principalement dans le milieu associatif à Halifax et dans le sud-ouest de la province depuis 2003. Dans ce contexte, nous avons mené des enquêtes en procédant notamment à de l'observation participante. Nous avons aussi eu recours à des documents écrits tels que des documents gouvernementaux, des articles dans des revues spécialisées, des journaux. Les liens que nous avons établis entre des thèmes comme langue, culture et santé nous ont conduite finalement à proposer quelques questions de recherche et quelques hypothèses de travail.
Tous droits réservés @ Université Sainte-Anne, 2010

Ce document est protégé par la loi sur le droit d'auteur. L'utilisation des services d'Érudit (y compris la reproduction) est assujettie à sa politique d'utilisation que vous pouvez consulter en ligne.

https://apropos.erudit.org/fr/usagers/politique-dutilisation/ 


\title{
Repérage de quelques obstacles linguistiques et culturels des soins de santé pour les immigrants francophones de la Nouvelle-Écosse ${ }^{1}$
}

\author{
Louise Fontaine \\ Université Sainte-Anne
}

\begin{abstract}
Résumé
Ce texte s'attarde au concept d'“ immigrant francophone » appliqué au contexte de la Nouvelle-Écosse. Contrairement à ce qu'on peut imaginer à un premier niveau d'analyse, ce concept est polysémique et le sens qu'on y attribue varie selon les acteurs sociaux qui l'utilisent. Donc, afin de mieux délimiter l'objet de notre propos, nous avançons quelques éléments de définition à prendre en compte. Il s'agit d'une recherche exploratoire orientée vers la formulation de quelques pistes de réflexion, voire quelques hypothèses de travail à vérifier lors de la réalisation de futures recherches dans le domaine de la santé auprès des communautés francophones et acadiennes de la Nouvelle-Écosse. Pour étoffer notre propos, nous présentons quelques données empiriques recueillies à même le travail de terrain mené principalement dans le milieu associatif à Halifax et dans le sud-ouest de la province depuis 2003. Dans ce contexte, nous avons mené des enquêtes en procédant notamment à de l'observation participante. Nous avons aussi eu recours à des documents écrits tels que des documents gouvernementaux, des articles dans des revues spécialisées, des journaux. Les liens que nous avons établis entre des thèmes comme langue, culture et santé nous ont conduite finalement à proposer quelques questions de recherche et quelques hypothèses de travail.
\end{abstract}

Le Canada a été et est encore une terre d'accueil pour de nombreuses personnes qui sont très diversifiées du point de vue ethnique ${ }^{2}$ et qui proviennent de divers horizons géographiques ${ }^{3}$. Cet état de fait, qui est largement admis, a donné lieu à l'élaboration de multiples désignations pour qualifier cette fraction de la population qui est née à l'extérieur du Canada. Si on veut bien saisir les enjeux entourant l'immigration internationale, il s'avère important de bien identifier les énoncés de principe

1. La version préliminaire de ce texte a été présentée lors du $2^{\mathrm{e}}$ Forum national de recherche sur la santé des communautés francophones en situation minoritaire Société, langue et santé : les barrières à l'accès aux services de santé pour les communautés francophones minoritaires, à Ottawa, le 23 novembre 2007.

2. Tout comme le soutient Max Weber, ce qualificatif correspond à une croyance subjective à une communauté d’origine, qu'elle soit réelle ou imaginaire. Cette «communauté d'origine », qui est tantôt définie à partir d'une identité religieuse, linguistique ou culturelle, encadre et structure la vie sociale des individus. Voir Louise Fontaine, "Le quadrillage ethnique de Bruxelles », dans Recherches sociologiques, 1999, vol. xxx, n 1, p. 118.

3. Jacques Henripin, "Chapitre 12 - Les migrations ", dans La métamorphose de la population canadienne, Montréal, Les Éditions Varia, " Histoire et société », 2003, p. 209-237. 
élaborés par les acteurs étatiques (dirigeants politiques, fonctionnaires ou autres), analyser l'application concrète de ces principes d'action par des représentants d'associations communautaires qui agissent notamment en tant que relais de la sphère politique et enfin explorer plus à fond ce qui se vit au jour le jour par des individus, notamment des immigrants francophones qui s'établissent dans notre voisinage et que l'on rencontre à l'hôpital, à l'épicerie, à l'école, dans leur milieu de travail, etc.

Ce sont principalement ces dimensions de la vie sociale que nous allons privilégier dans notre texte. Soulignons que notre contribution sera plutôt modeste par rapport à l'analyse des soins de santé offerts à une fraction particulière de la population néo-écossaise que nous désignons en tant qu'immigrants francophones. Ce fait se justifie, car, depuis 2003, nos recherches se concentrent surtout sur l'immigration en Nouvelle-Écosse d'un point de vue global. Toutefois, il s'avère pertinent de repérer quelques obstacles linguistiques et culturels rencontrés par des immigrants francophones lorsqu'ils cherchent à obtenir des soins de la part des professionnels de la santé dans la province. Nous nous proposons donc de partager quelques découvertes de terrain en partant de la question suivante : comment peut-on relier ensemble les quatre éléments - langue, culture, soin de santé et immigrant francophone - dans le contexte néo-écossais?

\section{Quelques balises théoriques}

La notion de francophonie soulève toute une série de considérations, car, contrairement à ce qu'on peut imaginer à un premier niveau d'analyse, ce concept est polysémique et le sens qu'on lui attribue varie selon les acteurs sociaux qui l'utilisent. Tantôt la langue française fait référence à la langue maternelle, tantôt elle peut être associée à la première langue apprise, la langue d'usage à la maison, au travail, à l'école... Ce terme recouvre une réalité plutôt complexe à délimiter.

Il n’y a pas unanimité en ce qui concerne la définition objective de cette notion, notamment dans le domaine de la démolinguistique. Certains prennent en compte la question de la langue maternelle, d'autres celle de la langue parlée à la maison, du pays d'origine, de la langue de l'enseignement, [la langue de travail], etc. ${ }^{4}$

4. Office québécois de la langue française, "Francophone », dans le Grand dictionnaire terminologique, 2003 [en ligne], www.granddictionnaire.com (page consultée le 3 mars 2010). 
Carsten Quell met en lumière ce type de problème lorsqu'il pose la question : «Qui est considéré comme francophone? » À cela, il répond :

Au Canada, c'est habituellement la langue maternelle qui détermine si une personne est francophone ou anglophone. Cela pose toutefois problème pour un nombre de plus en plus grand de nouveaux arrivants. En effet, de nombreux immigrants francophones ont comme langue maternelle l'arabe, le créole, le lingala, le somali ou le tchilouba, mais ont fait leurs études et travaillent en français. En réalité, la plupart de ces immigrants se considèrent francophones. Cela étant, si on définit les francophones uniquement sur la base de la langue maternelle, on ne tient pas compte de la complexité de la réalité linguistique actuelle. ${ }^{5}$

C'est pourquoi, et malgré le fait que Statistique Canada utilise souvent la langue maternelle comme critère principal pour définir un francophone, pour les fins de la présente étude, nous retenons qu'un ou une francophone désigne " une personne qui utilise couramment la langue française dans la plupart des situations de la vie quotidienne ${ }^{6}$.

Pour ce qui est du concept d'immigrant, il correspond à tout individu qui répond aux deux conditions suivantes : il est né à l'extérieur du Canada et il s'y est assez récemment établi, étant entendu que cette période d'établissement dépasse douze mois de résidence permanente; sinon, tout comme le Gouvernement du Canada, on parlerait alors de nouvel arrivant ${ }^{7}$. Autrement dit, nous ne nous attarderons pas sur les personnes établies depuis plus d'une génération, sachant que le statut d'immigrant est un statut transitoire.

Le terme d'immigrant francophone désigne alors tout individu qui répond à ces deux conditions et qui fait usage de la langue française dans la plupart des situations qu'il vit au quotidien. La langue maternelle n'est donc pas un critère que nous retenons ici. Nous préférons nous intéresser à la langue d'usage. De plus, signalons que, dans le cas de la Nouvelle-Écosse, l'usage de la langue française dans la plupart des situations sociales revêt un caractère très particulier et souvent beaucoup plus

5. Carsten Quell, L'immigration et les langues officielles : obstacles et possibilités qui se présentent aux immigrants et aux communautés, Commissariat aux langues officielles, Ministère des Travaux publics et Services gouvernementaux Canada, novembre 2002, p. 9.

6. Office québécois de la langue française, loc. cit.

7. Louise Fontaine, L'immigration francophone en Nouvelle-Écosse, document d'information (en collaboration avec la Fédération acadienne de la Nouvelle-Écosse (FANE) et Citoyenneté et immigration Canada (CIC)), avril 2005, p. 47 [en ligne], «http:// www.ssta.org/media_uploads/pdf/3899.pdf > (document consulté le 3 mars 2010). 
complexe qu'on ose le dire. En fait, si on fait abstraction des dimensions politiques et de la charge émotive associées à la langue française, en Nouvelle-Écosse, la plupart des interactions sociales se déroulent simultanément tantôt en français "standard », tantôt en anglais, et aussi en acadien $^{8}$.

Pour ce qui est du concept de culture, tout comme le souligne Amin Maalouf,
[...] nous sommes tous infiniment plus proches de nos contemporains que de nos ancêtres. [...] Non seulement dans l'aspect, dans le vêtement, dans la démarche, non seulement dans le mode de vie, le travail, l'habitat, les instruments qui nous entourent, mais aussi dans les conceptions morales, dans les habitudes de pensée. ${ }^{9}$

Pour sa part, Georges Balandier soutient qu'il a "naguère tenté de faire l'inventaire des définitions du concept, [...et qu'il en a] recensé plus de $150 »^{10}$. En tenant compte de tous les éléments, retenons que le concept de culture renvoie à un ensemble
de symboles, de croyances et de divers codes de communication qui sont partagés par un certain nombre d'individus. Il s'agit de divers attributs, des dénominateurs communs entre des individus qui se reconnaissent à tort ou à raison des liens d'appartenance fondés sur la langue, le territoire, la religion, la culture, l'origine ethnique, la mémoire collective... Ces critères définissent des systèmes de classification pour les individus qui se retrouvent sur des territoires juridiquement reconnus. ${ }^{11}$

Pour ce qui est du concept de soins de santé, celui-ci désigne l'« ensemble des soins offerts à chaque membre d'une collectivité par des professionnels des services de santé et comprenant les soins médicaux, la

8. Id., p. 17.

9. Amin Maalouf, Les identités meurtrières, Paris, Éditions Grasset \& Fasquelle, 2008, p. 136.

10. Georges Balandier, Civilisés, dit-on, Paris, Presses Universitaires de France, 2003, p. 314 .

11. Louise Fontaine, «Confusions identitaires : quelques observations provenant de divers acteurs étatiques », dans Carol J. Harvey et Alan MacDonell [dir.], La francophonie sur les marges, actes du seizième colloque du Céfco (Centre d'études francocanadiennes de l'Ouest, 17-19 octobre 1996), Winnipeg, Presses Universitaires de Saint-Boniface, 1997, p. 27. 
réadaptation, le dépistage, la prévention de la maladie ainsi que l'éducation sanitaire $»^{12}$.

Ces définitions, qui délimitent quelque peu notre propos, nous invitent maintenant à formuler les questions de recherche suivantes : Quels mots choisir pour s'exprimer en langage clair et être compris? Quel niveau de langage utiliser avec son interlocuteur? Comment surmonter la rivalité historique entre " anglophones » et "francophones " pour désigner telle ou telle maladie et comprendre le patient et être compris par ce dernier?

\section{Méthodes de recherche utilisées}

Notre travail de terrain au sein de l'Association métropolitaine pour l'établissement des immigrants (АмÉl)/Metropolitan Immigrant Settlement Association (MISA) ${ }^{13}$ s'est étalé sur une période de dix mois, soit de septembre 2003 à juin 2004, à raison de quelques jours par mois. Nous avons passé un total de vingt-quatre jours dans cette association. Pour la collecte de nos données, nous avons conjugué plusieurs méthodes de recherche : l'observation directe, l'observation participante, des entrevues semi-structurées, des discussions formelles et de nombreuses rencontres informelles avec des membres de cette association, qu'ils soient employés, bénévoles ou clients. À cela s'ajoute une analyse de plusieurs documents officiels, tels que les rapports annuels de cette association, des dépliants, des manuels élaborés par des employés, des feuilles distribuées dans des ateliers organisés au sein de cette association, des brochures publiées ou disponibles en ligne qui présentent les services offerts aux « immigrants » dans la ville d'Halifax, en Nouvelle-Écosse ou au Canada. Tout comme Howard S. Becker le formulait : "Au début, le chercheur peut ne pas être sûr du problème le plus intéressant à étudier dans la communauté ou l'organisation sur laquelle il travaille; il consacre ses premiers efforts analytiques à dégager des problèmes et des hypothèses les plus utiles pour les attaquer. $^{14}$

Notre analyse actuelle va dans ce sens, car elle n'a pas d'autre ambition que d'identifier des avenues de recherche à examiner ultérieurement dans des recherches plus approfondies, lorsqu'on met en relation des

12. Office de la langue française, "Soins de santé ", dans Grand dictionnaire terminologique, 2001 [en ligne], www.granddictionnaire.com (page consultée le 3 mars 2010).

13. Depuis le $1^{\text {er }}$ décembre 2009 , cet organisme est devenu Immigrant Settlement \& Integration Services (IsIS). L'Amél/MISA a fusionné avec une autre association : Halifax Immigrant Learning Centre (HILC). Cet autre organisme offre aussi des services aux nouveaux arrivants à Halifax et ailleurs en Nouvelle-Écosse.

14. Howard S. Becker, Le travail sociologique - Méthode et substance, Fribourg, Academic Press Fribourg, Éditions Saint-Paul, « Res socialis », 2006, p. 121. 
variables comme la langue, la culture, les soins de santé et les immigrants francophones établis au Canada. Parmi les hypothèses que nous pouvons formuler, il y a celle où nous soutenons que les immigrants francophones de la Nouvelle-Écosse semblent rencontrer les mêmes types de difficultés dans leurs relations avec les professionnels de la santé ${ }^{15}$ que les autres catégories de la population en général, mais ces difficultés peuvent être exacerbées dans certains cas.

De plus, il semble qu'il est quasi inévitable d'avoir une assez bonne connaissance de l'anglais ${ }^{16}$ pour pouvoir vivre et résoudre de nombreux problèmes liés au quotidien en Nouvelle-Écosse, toutes origines nationales ou ethniques confondues ${ }^{17}$.

Il est probant que, dans le cas notamment d'immigrants francophones établis en Nouvelle-Écosse, le patient arrive avec des symptômes d'une maladie inconnue au Canada et que le médecin ou les autres professionnels de la santé qui traitent ce cas ne connaissent pas de manière précise les détails de ce problème de santé. Pensons par exemple à la leptospirose, à la malaria, à la fièvre dingue, à la maladie de Chagas et à la leishmaniose.

Sachant que la plupart des immigrants francophones sont des travailleurs qualifiés détenteurs d'un diplôme universitaire, ils ont peut-être tendance à remettre en question l'autorité du médecin ou autre professionnel de la santé qui les traite, car un assez grand nombre d'utilisateurs de l'Internet vérifient et remettent en question la validité de leur diagnostic.

15. Les «médecins et les infirmières représentent un peu moins de 50 pour cent de l'ensemble des travailleurs de la santé. Les autres viennent d'une gamme étendue de professions ». Voir Institut canadien d'information sur la santé, Les soins de santé au Canada 2007, Ottawa, Icıs, 2007, p. 16 [en ligne], «http://secure.cihi.ca/cihiweb/ products/hcic2007_f.pdf> (document consulté le 27 mars 2008).

16. Cette remarque est à nuancer en ce sens qu'en août 2009 , la ministre de la Santé a officiellement annoncé l'instauration d'un service Info-Santé 811 qui « sera offert en plusieurs langues grâce à des infirmières francophones et à un tiers fournisseur de services de traduction sur demande ». Voir "Lancement du service Info-Santé 811 en Nouvelle-Écosse », Le Courrier de la Nouvelle-Écosse, vendredi 7 août 2009, p. 4.

17. Elsa Ramos, L'invention des origines - Sociologie de l'ancrage identitaire, Paris, Armand Colin Éditeur, "Sociétales », 2006, 220 p. 


\section{L'immigration en Nouvelle-Écosse}

Le 28 juin 2002, le gouvernement du Canada adopte la Loi sur l'immigration et la protection des réfugiés. Ce cadre juridique propose un système de classement de la population nouvellement arrivée au Canada. Parmi les catégories officiellement définies, on distingue principalement les résidents permanents et les résidents temporaires. Les résidents permanents sont définis de manière plus spécifique à partir de quatre catégories particulières, soit l'immigration économique, le regroupement familial, les réfugiés, les autres immigrants. Le statut de résident temporaire renvoie aussi à quatre catégories particulières : les étudiants étrangers, les travailleurs étrangers, les cas humanitaires et les autres résidents temporaires ${ }^{18}$. Cette catégorisation officielle du gouvernement central à Ottawa, qui s'applique dans la province, a donné lieu à l'élaboration d'une politique d'immigration provinciale qui a été formalisée en janvier 2005. C'est le moment où le gouvernement de la Nouvelle-Écosse a officiellement annoncé un cadre stratégique en matière d'immigration pour la province ${ }^{19}$.

Pour l'année 2006, la population totale de la Nouvelle-Écosse se chiffre à $913462^{20}$. De ce nombre, 372679 personnes vivent à Halifax soit 40,8 pour cent ${ }^{21}$. À titre indicatif, pour cette même année, la NouvelleÉcosse a admis 2585 résidents permanents sur un total de 251649 résidents permanents admis pour l'ensemble du Canada, soit 1 pour cent ${ }^{22}$. Le total de ces 2585 résidents permanents se répartit de la manière suivante : 1916 résidents permanents habitent Halifax et les 669 autres habitent dans le reste de la province, ce qui correspond à 74 pour cent du nombre total pour la région métropolitaine d'Halifax ${ }^{23}$.

Par ordre d'importance, les résidents permanents dans la province se subdivisent de la manière suivante : dans la catégorie de l’immigration

18. Citoyenneté et immigration Canada, Canada Atlantique - Aperçu de l'immigration / Résidents permanents et temporaires - Faits et chiffres 2006, 2007, 68 p.

19. Communications Nova Scotia, Cadre stratégique pour l'immigration, document de travail, août 2004, 54 p.; id., Stratégie de la Nouvelle-Écosse en matière d'immigration, janvier 2005, 35 p.

20. Statistique Canada, Profils des communautés de 2006, "Faits saillants pour la communauté de Clare », 2007, date de modification 09-12-2007 [en ligne], 〈http:// www12.statcan.cas (document consulté le 26 octobre 2007).

21. Ministère des Finances de la Nouvelle-Écosse, Division des statistiques, 2006 Census of Canada Nova Scotia Perspective, 2006, p. 3 [en ligne], shttp://www.gov. ns.ca/finance/statistics/agency/publications/Cen2006.pdf) (document consulté le 20 novembre 2007).

22. Citoyenneté et immigration Canada, Faits et chiffres 2006 - Aperçu de l'immigration / Résidents permanents et temporaires, 2007, p. 3 [en ligne], «http://www.cic. gc.ca/francais/pdf/pub/faits2006.pdf) (document consulté le 5 mars 2008).

23. Id., p. 38 . 
économique, on retrouve 1774 personnes (68,6 pour cent), pour ce qui est du regroupement familial, on dénombre 510 personnes (19,7 pour cent), les réfugiés sont au nombre de 217 (8,4 pour cent) et pour ce qui est de la catégorie " autres immigrants », on comptabilise 84 personnes (3,2 pour cent) ${ }^{24}$.

Pour ce qui est des connaissances linguistiques des résidents permanents de la Nouvelle-Écosse, le tableau 1 dresse un portrait succinct de la situation. On y observe que, malgré le fait que les connaissances linguistiques en français pour les résidents permanents ont augmenté entre 2005 et 2006 , le nombre total de ces individus demeurent très faible.

Tableau 1

\begin{tabular}{|l|r|r|}
\hline $\begin{array}{l}\text { Connaissances linguistiques pour les } \\
\text { résidents permanents de la N.-É. }\end{array}$ & $\mathbf{2 0 0 5}$ & \multicolumn{1}{|c|}{$\mathbf{2 0 0 6}$} \\
\hline anglais & 1301 & 1762 \\
\hline français & 13 & 22 \\
\hline anglais et français & 192 & 162 \\
\hline ni l'un ni l'autre & 423 & 639 \\
\hline TOTAL & 1929 & 2585 \\
\hline \multicolumn{2}{|c|}{ Source : Citoyenneté et immigration Canada, } \\
Faits et chiffres 2006 (cf. note 22)
\end{tabular}

Selon l'Office de l'immigration de la Nouvelle-Écosse, la provenance géographique des résidents permanents de la Nouvelle-Écosse en 2006 se présente de la manière suivante ${ }^{25}$ : les États-Unis, la Chine et le Royaume-Uni occupent respectivement les trois premières positions. Le tableau 2 présente ces données en spécifiant par ordre d'importance les sept autres pays d'origine pour un total de dix.

24. Id., p. 40-41.

25. Joëlle Désy, qui travaille à l'Office de l'immigration de la Nouvelle-Écosse, a présenté ses données statistiques lors de l'ouverture officielle d'une conférence qui s'est tenue à l'Université Sainte-Anne, le 2 novembre 2007. Cette conférence avait pour titre «Comment relever les défis de la régionalisation de l'immigration en milieu rural? ». 
Tableau 2

\begin{tabular}{|l|r|}
\hline $\begin{array}{l}\text { Pays d'origine en } \mathbf{2 0 0 6} \\
\text { (les dix premiers pays) }\end{array}$ & \multicolumn{1}{l|}{$\%$} \\
\hline États-Unis & $9,2 \%$ \\
\hline Chine & $8,2 \%$ \\
\hline Royaume-Uni & $8,0 \%$ \\
\hline Iran & $7,3 \%$ \\
\hline Corée & $6,2 \%$ \\
\hline Taïwan & $4,8 \%$ \\
\hline Inde & $4,1 \%$ \\
\hline Philippines & $3,6 \%$ \\
\hline Jordanie & $2,9 \%$ \\
\hline Liban & $2,4 \%$ \\
\hline
\end{tabular}

Source : Joëlle Désy, Office de l'immigration de la Nouvelle-Écosse, 2 novembre 2007 (cf. note 25)

\section{Quelques découvertes de terrain}

Plusieurs obstacles semblent se dresser pour l'immigrant francophone qui s'établit en Nouvelle-Écosse ${ }^{26}$. Ces obstacles varient selon le niveau d'études de cet individu en particulier. À cela, s'ajoute son niveau de compétences linguistiques en français et en anglais face à l'utilisation de termes techniques. Par exemple, on fait usage du mot stroke pour désigner un accident cérébro-vasculaire (ACV), diabetes pour parler de diabète, rabies pour la rage, AIDs pour parler du sida, flu pour parler d'une grippe, bird flu pour la grippe aviaire, cancer pour faire référence à un cancer. Ces éléments représentent en condensé la variété linguistique à laquelle peut être éventuellement confronté un immigrant francophone lorsqu'il est engagé dans une conversation, notamment avec un professionnel de la santé en Nouvelle-Écosse.

Des obstacles d'ordre culturel peuvent aussi se présenter. La problématique de la diversité linguistique et culturelle renvoie à des dimensions ethniques, religieuses, aux rapports entre les hommes et les femmes, aux manières de se représenter l'autorité médicale, à la perception de son corps, à la dénomination des parties de ce dernier et même à la conception de la vie dans sa globalité, et ce, jusqu'à son dernier souffle.

À plusieurs reprises, nous avons empiriquement observé que le fait d'émigrer engendre tantôt du stress tantôt un ensemble de symptômes qui sont nombreux et variés et qui recoupent l'univers de ce que

26. Louise Fontaine, L'immigration francophone en Nouvelle-Écosse, op. cit., p. 18. 
certains nomment les maladies psychosomatiques. Le projet d'émigrer et une situation sans emploi une fois établie en Nouvelle-Écosse perturbent l'équilibre de plusieurs couples et familles. En plus, il y a aussi ce qu'on peut désigner comme étant le désenchantement après environ un an d'établissement, car des services d'accompagnement ou des réseaux d'entraide ne sont pas nécessairement constitués. À titre indicatif, pour la période 1991-2001, seulement 38 pour cent du nombre total des nouveaux arrivants accueillis dans la province y sont restés ${ }^{27}$. Que faire pour résoudre des questions comme : qui peut garder mes enfants pendant que je vais voir le dentiste ou un médecin, etc.?

Un autre exemple significatif est celui relatif à l'affichage dans la salle d'attente d'un médecin et dans son cabinet. L'affichage en anglais prédomine. Ce phénomène a aussi été observé dans les salles de classe prévues pour la formation en soins ambulanciers à l'Université SainteAnne. Notons qu'en août 2007, nous avons suivi une formation d'un jour sur le secourisme. Le manuel distribué était en français dit « standard », le cours a été présenté surtout en acadien et il s'adressait à toutes les catégories d'employés de l'Université Sainte-Anne. À nos côtés, une employée de soutien (établie en Nouvelle-Écosse depuis de nombreuses années et fort probablement née dans la province) a témoigné à la fin de la formation en précisant qu'elle n'avait pas compris ce qui était présenté à l'écran ou sur les feuilles distribuées.

Parmi les initiatives visant à développer des services de santé en langue française, soulignons que l'Université Sainte-Anne a été approchée en 2005 afin de proposer un cours relatif à The Cultural Health Information and Interpreting Service (CHI-IS), cours qui avait été offert au Nova Scotia Community College dans le cadre d'un projet pilote. Cette proposition n'a pas connu d'aboutissement concret. Toutefois, l'Université Sainte-Anne offre cinq programmes d'études postsecondaires en français dans le domaine de la santé, grâce à l'appui du Consortium national de formation en santé ${ }^{28}$.

Le site Web de l'Office de l'immigration de la Nouvelle-Écosse parle d'un service d'interprète linguistique et culturel, mais la langue française ne semble pas retenir particulièrement l'attention au sein de cet organisme gouvernemental ${ }^{29}$. Lors de notre travail de terrain à Halifax en

27. Metropolitan Immigrant Settlement Association, Report on the Nova Scotia Immigration Partnership Conference 2003 - Opportunities for Collaboration, July 2003 , p. 37.

28. Consortium national de formation en santé (CNFS) (2007/2008), Rapport annuel 2007/2008, p. 13 [en ligne], 〈http://www.cnfs.net/publications/rapports-annuels〉 (document consulté le 8 septembre 2008).

29. Office de l'immigration de la Nouvelle-Écosse, Health Interpretation Services, 2007 
2003-2004, nous avons été témoin de cette offre de services auprès de l'Association métropolitaine pour l'établissement des immigrants (Amél) ${ }^{30}$. Cette organisation a préparé un document qui présente différents types d'interprétation culturelle, notamment par rapport aux soins de santé et en ce qui a trait à la santé mentale ${ }^{31}$. Signalons que cette organisation non gouvernementale élargit son offre de services de première ligne en région en ayant recours aux nouvelles technologies de l'information et des communications ${ }^{32}$.

Les services d'accueil et d'établissement qui sont offerts aux nouveaux arrivants par un organisme de première ligne comme Amél sont fort complexes et multidimensionnels. Pour ce qui est du cas des réfugiés parrainés par le gouvernement (RPG), les services offerts à ces individus sont régis par des critères d'admissibilité définis par le gouvernement du Canada. Celui-ci finance notamment le programme d'aide au réétablissement (PAR) et le programme d'établissement et d'adaptation des immigrants (PÉAI). La première année d'établissement à Halifax se divise en trois étapes : durant les six premières semaines; après trois mois d'établissement; après neuf mois. Dès le lendemain de leur arrivée à Halifax, les nouveaux arrivants sont invités à une session d'orientation lors de laquelle une trousse d'information leur est remise. Parmi les informations qui y sont rassemblées, il y a un dépliant relatif aux mesures de sécurité à prendre à la maison, un document préparé par l'Institut pour la prévention de l'enfance maltraitée et des informations en ce qui a trait aux droits du patient dans un hôpital33.

Outre ces démarches d'accompagnement, l'AmÉl offre aussi un suivi individualisé auprès de cette catégorie de réfugiés à partir d’une formule assez souple. Par exemple, une fois par semaine, un employé se présente à l'école ou dans les lieux où sont offerts des cours d'anglais langue seconde, des cours financés par le gouvernement du Canada et conçus pour cette clientèle particulière. Le moment choisi est un peu avant midi,

[en ligne], 〈http://www.novascotiaimmigration.com/en-page1062.aspx〉 (document consulté le 27 mars 2008).

30. Revoir la note 13.

31. Merek Jagielski, Cultural Interpretation Handbook, Halifax, Metropolitan Immigrant Settlement Association, 2003, p. 50-53.

32. Ressources humaines et développement social Canada, Travailler avec les immigrants en dehors de la Municipalité régionale d'Halifax (MHR) / Work in Nova Scotia - A bridging program for newcomers, rapport final. Initiative de développement à distance des compétences en milieu de travail HILC [Halifax Immigrant Learning Centre] et AmÉl-MISA, juin 2007, 51 p.

33. Louise Fontaine, "Processus d'établissement, nouvel arrivant et structure d'accueil à Halifax (Nouvelle-Écosse) : une exploration de quelques actions concrètes », dans Canadian Ethnic Studies/Études ethniques au Canada, 2005, vol. xxxvII, $\mathrm{n}^{\circ} 3$, p. 142 . 
de sorte que ces étudiants adultes peuvent s'adresser à cet employé durant l'heure du midi pour obtenir des informations particulières face à un problème qu'ils rencontrent. Ces discussions se déroulent de manière informelle parfois même dans les corridors de l'école. Nous avons fait de l'observation directe pendant que de telles situations particulières se déroulaient à l'école Queen Elizabeth High. Par exemple, on a tenté de résoudre des problèmes médicaux, un problème de visite chez le dentiste et un problème relatif aux remboursements autorisés par le gouvernement fédéral, etc. ${ }^{34}$.

Parmi les autres sources d'information disponibles pour les immigrants francophones, signalons le site Web lancé par le Comité directeur de l'immigration francophone de la Nouvelle-Écosse, comité formé de représentants de six organismes, dont la Fédération acadienne de la NouvelleÉcosse (FANE). Ce site s'articule autour de trois thèmes principaux : venez, découvrez et restez. Sous cette dernière rubrique, on définit des étapes à suivre pour s'établir dans la province ${ }^{35}$. Signalons qu'en 2006, la FANE a organisé une tournée dans les diverses régions acadiennes et francophones de la province afin de promouvoir l'immigration francophone en Nouvelle-Écosse. Deux ans plus tard, les résultats demeurent plutôt limités ${ }^{36}$.

Il est aussi possible de consulter en ligne un Répertoire des fournisseurs de soins de santé primaires francophones pour la Nouvelle-Écosse. Il est disponible sur le site du ministère de la Santé37. Mentionnons aussi de nombreuses "ressources en soins de santé » proposées par le gouvernement de la Nouvelle-Écosse dans une rubrique qui a pour titre "Événements de la vie "38.

Rappelons que notre travail de terrain, réalisé depuis 2003 à Halifax et aussi dans le sud-ouest de la province ${ }^{39}$, a impliqué notamment de

34. Id., p. 144 .

35. L’Acadie de la Nouvelle-Écosse, Accueil, 2009 [en ligne], «http://www.vienouvelleecosse.cas (document consulté le 3 mars 2010).

36. Martin Paquet, "L'immigration francophone en Nouvelle-Écosse », dans Canadian Issues/Thèmes canadiens, Chedly Belkhodja [directeur invité], "Immigration et diversité dans les communautés francophones en situation minoritaire », printemps 2008, p. 99-103.

37. Province de la Nouvelle-Écosse, Répertoire des fournisseurs de soins de santé primaires francophones, 2008 [en ligne], 〈http://www.gov.ns.ca/health/frhcp/ default.asp» (document consulté le 27 mars 2008).

38. Province de la Nouvelle-Écosse, Ressources de soins de santé, 2007 [en ligne], 〈http://www.gov.ns.ca/snsmr/lifeevents/f/fr-default.asp〉 (document consulté le 20 novembre 2007 ).

39. Louise Fontaine, "L'immigration rurale et francophone en Nouvelle-Écosse : quelques pistes de réflexion », dans Canadian Issues/Thèmes canadiens, Chedly Belkhodja [directeur invité], «Immigration et diversité dans les communautés fran- 
mener des enquêtes en procédant à des observations participantes et à analyser des documents gouvernementaux, des articles dans des revues spécialisées et dans des journaux. Par rapport à la problématique qui nous intéresse ici, il ressort que, parmi les obstacles observés, figurent le degré de scolarité, le niveau de compétence en anglais et en français par rapport à la terminologie médicale, la perception de l'autorité exercée par les professionnels de la santé, l'image de son propre corps et sa conception de la vie et de la mort. Ces pistes présentées ici ouvrent la voie à des recherches plus approfondies. Notre étude n'a fait que repérer un certain nombre d'obstacles linguistiques et culturels à surmonter pour une fraction de la population de la Nouvelle-Écosse, qui se retrouve en situation minoritaire, car cette province, qui n'est pas officiellement bilingue, continue d'avoir une population «francophone » qui oscille entre 3 pour cent et 5 pour cent de sa population totale.

cophones en situation minoritaire ", printemps 2008, p. 79-82. 